\title{
Academy of Dental Materials guidance on in vitro testing of dental composite bonding effectiveness to dentin/enamel using micro-tensile bond strength ( $\mu \mathrm{TBS})$ approach
}

\author{
Steve Armstrong ${ }^{a, *}$, Lorenzo Breschi ${ }^{b}$, Mutlu Özcan $^{c}$, Frank Pfefferkorn ${ }^{d}$, \\ Marco Ferrari ${ }^{e}$, Bart Van Meerbeek ${ }^{f}$ \\ a Department of Operative Dentistry, College of Dentistry and Dental Clinics, University of Iowa, USA \\ ${ }^{\mathrm{b}}$ Department of Biomedical and Neuromotor Sciences, DIBINEM, University of Bolgna, Italy \\ c Dental Materials Unit, Center for Dental and Oral Medicine, Clinic for Rixed and Removable Prosthodontics and \\ Dental Materials Science, University of Zurich, Switzerland \\ d Dental Research, Clinical Affairs, Dentsply Sirona Restorative, Germany \\ e Department of Prosthodontics and Dental Materials, University of Siena, Italy \\ f Department of Oral Health Sciences, Catholic University of Leuven, Belgium
}

A R T I C L E I N F O

Article history:

Received 18 November 2016

Accepted 29 November 2016

Available online $\mathrm{xxx}$

Keywords:

Bonding

Micro-tensile

Dentin

Enamel

Composite

Bond strength

Laboratory

Guidance

Guidelines

\section{A B S T R A C T}

Objective. An ideal dental adhesive should provide retentive strength, marginal seal, be relatively simple to achieve and demonstrate clinical durability. Future improvements in adhesive bonding to tooth structure require in vitro test methods that provide reliable data for materials development and/or evaluation of experimental variables. The objective of this project was to identify a test method that is relatively easy to perform, repeatable and ultimately useful for predicting clinical outcomes.

Methods. The Academy of Dental Materials initiated a project to develop and distribute guidance documents on laboratory test methods that are useful for the evaluation of dental adhesives and cements, composite resins and ceramics.

Results. The dental adhesive sub-group has identified the micro-tensile bond strength test, especially after subjecting the specimens to a durability challenge, as currently the best practical surrogate measure of dental composite restoration retention.

Conclusion. The following $\mu$ TBS guidance is meant to aid the researcher in conducting the $\mu$ TBS test. The authors, while recognizing the limitations of a static, strength-based test method, welcome comments and suggestions for improvements of this guidance document in future revisions.

( 2016 The Academy of Dental Materials. Published by Elsevier Ltd. All rights reserved.

\footnotetext{
* Corresponding author.

E-mail addresses: steven-armstrong@uiowa.edu (S. Armstrong), lorenzo.breschi@unibo.it (L. Breschi), mutluozcan@hotmail.com (M. Özcan), Frank.Pfefferkorn@dentsplysirona.com (F. Pfefferkorn), ferrarm@gmail.com (M. Ferrari), bart.vanmeerbeek@med.kuleuven.ac.be (B. Van Meerbeek). http://dx.doi.org/10.1016/j.dental.2016.11.015 0109-5641/@ 2016 The Academy of Dental Materials. Published by Elsevier Ltd. All rights reserved.
} 
A

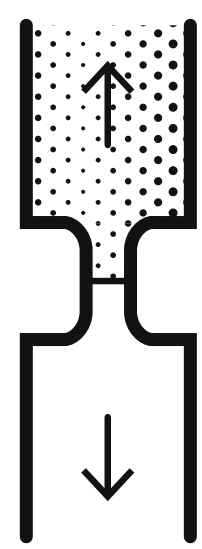

B

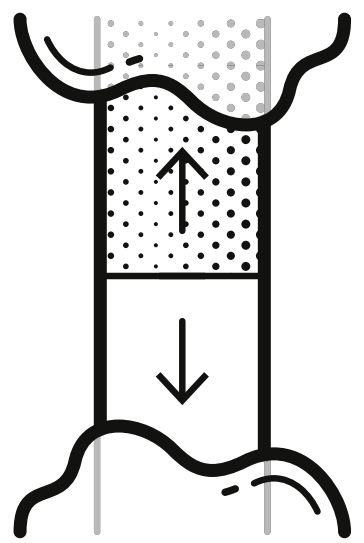

Fig. 1 - (A) Passive gripping device; (B) active gripping device.

\section{Introduction}

Future improvements in adhesive bonding to tooth structure require in vitro test methods that provide reliable data for materials development and/or evaluation of experimental variables. Multiple test methods are necessary for understanding and improving the clinical performance of dental materials; however, a chosen test method should be relatively easy to perform and must be repeatable within and between laboratories, and ultimately useful for predicting clinical outcomes [1]. Ideally, adhesion to tooth structure should provide retentive strength, marginal seal, be relatively simple to achieve and have clinical durability. The microtensile bond strength test ( $\mu \mathrm{TBS}$ ), especially after subjecting the specimens to a durability challenge, is currently recommended by the authors as the best surrogate measure of dental composite restoration retention [2]. The following guidelines are meant to aid the researcher in conducting the $\mu$ TBS test. The authors welcome comments and suggestions for improvements of guidelines in future revisions.

\subsection{Terminology [3]}

a) Passive gripping device (Fig. 1A): specimen is placed in a testing device without the aid of glue or mechanical gripping; device should self-align the specimen parallel to the tensile load.

b) Active gripping device (Fig. 1B): mechanical fastening of specimen to gripping device, such as with the use of glue or "clamps".

c) Stick specimen (Fig. 2A): cross-sectionally square microspecimen, also referred to as a "non-trimmed specimen" or sometimes as a "beam".

d) Dumbbell specimen (Fig. 2B): a smoothly notched specimen with a defined radius of curvature from the end or shoulder of the specimen to a straight gauge length that defines the testing region and includes the adhesive joint. This specimen is one kind of a "trimmed specimen". Although trimming by hand has been done, it is highly preferable to machine the dumbbells using mechanically or computer-
A

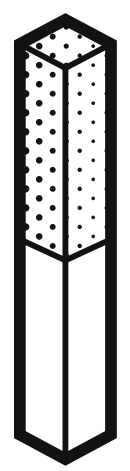

B

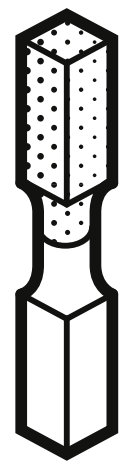

C

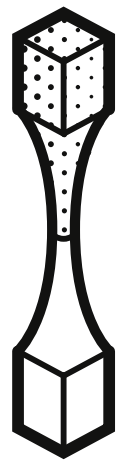

Fig. 2 - (A) Stick specimen; (B) dumbbell specimen; (C) hourglass specimen.

controlled high-speed hand piece movement to produce exacting geometries with identical fabrication histories (Fig. 3).

e) Hourglass specimen (Fig. 2C): a notched specimen with a defined radius of curvature from the end or shoulder of the specimen to the adhesive joint without a defined straight gauge length. This specimen is the other kind of a "trimmed" specimen.

f) Pre-testing failures ("ptf"): a specimen failure that occurred before tensile testing and cannot be attributed to any human manipulation error (see below); it possesses an unknown bond strength, which is an observation commonly referring to weak adhesive performance and therefore cannot be ignored; it has also been referred to as left-censored data.

g) Manipulation error: a specimen failure that occurred at any moment during specimen preparation or testing and should be attributed to a human manipulation error and should be clearly differed from the abovementioned pretesting failures. Occurrence and number needs to be explicitly noted, but are excluded from the dataset.

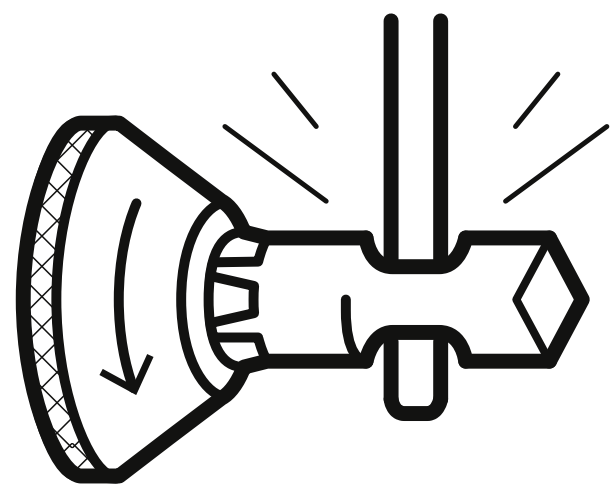

Fig. 3 - Schematic illustrating the preparation of a 'trimmed' dumbbell specimen. Dumbbell micro-specimens are preferentially prepared using mechanically or computer-controlled high-speed hand piece movement, e.g. MicroSpecimen Former (University of Iowa). 


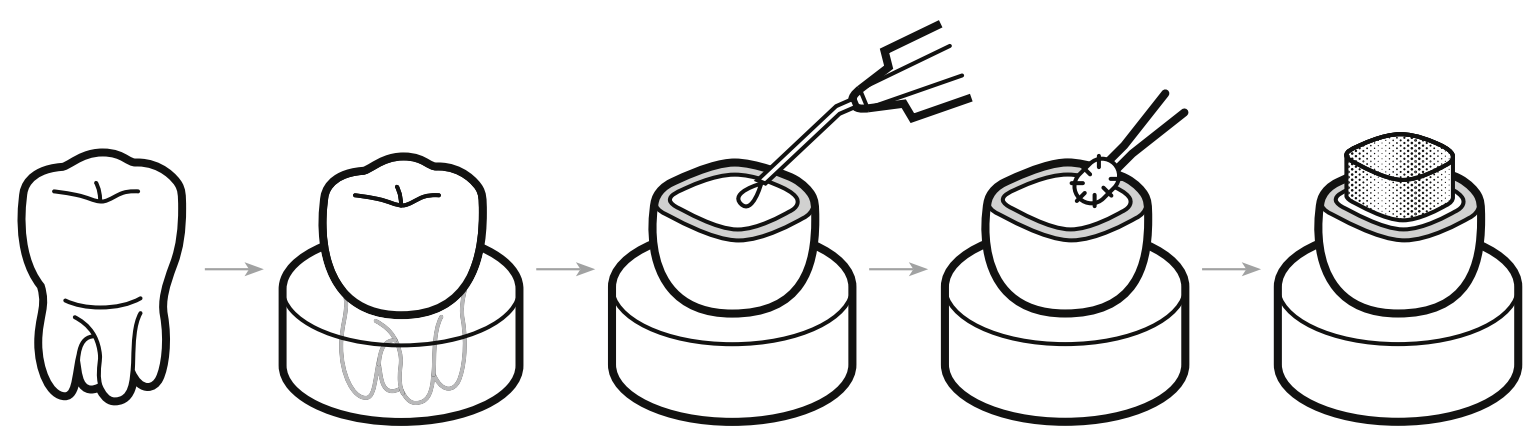

Fig. 4 - Schematic illustrating the successive steps of tooth mounting and bonding.

h) Tooth dependency: when testing multiple specimens from the same tooth. Tooth dependency must be accounted for if each specimen is considered as an experimental unit, e.g. random effects for ANOVA, frailty effects for Weibull [4]. Seek expert statistical support.

\section{Guidelines/specific recommendations for adhesive-dentin $\mu$ TBS testing (recommended approach highlighted in bold when multiple options are given)}

1. Selection of the teeth:

a) Ethics: Project must comply with local Medical Ethics Committee requirements. Local Medical Ethics Committees may grant an exemption for formal review when using teeth that have been discarded as medical waste material and recuperated for secondary use without patient identifiers.

b) Substrate: Human permanent molars (or premolars) (deciduous teeth if required by research question).

c) Age of teeth: should be described if available, but is essential if age of substrate is the variable to be investigated.

Note: "It is preferable to use third permanent molars from 16- to 40-year-old individuals if possible" (according to ISO/DTS 11405 Dentistry - Testing of adhesion to tooth structure) [5].

d) Number of teeth: Ideally based upon power calculation performed in planning stages of study; if the specimen is used as the statistical unit, an absolute minimum of three teeth per experimental group with appropriate consideration of tooth dependency/clustering; if tooth is the statistical unit at least 5 and by preference $8-10$ teeth per experimental group.

e) Post-extraction time: preferable less than 1 month. Note: less than 6 months according to ISO/DTS 11405 [5].

f) Condition of teeth: non-restored, not root-canal treated, caries free, intact in area of bonding interest and ultimate test specimen geometry.

g) Additional substrate conditions: determined by research questions and research design, e.g. cariesaffected, sclerotic, erosive dentin, etc.

2. Storage of extracted teeth: a) Upon extraction: Store teeth in $0.5 \%$ Chloramine-T immediately after extraction.

b) Upon receipt in the laboratory: Remove adherent tissue, clean teeth and place in fresh $0.5 \%$ Chloramine- $\mathrm{T}$ and store at $4-7^{\circ} \mathrm{C}$.

c) Tooth hydration: Do not allow the tooth to dehydrate from point of extraction throughout the entire experimental process (continuously immerse in aqueous media without antimicrobial agent or cover with wet tissue paper in between the processing steps).

d) Aqueous storage media type: Laboratory grade water is not necessary, but if using deionized water be aware that tooth surface demineralization has been documented, therefore a phosphate-buffered salt solution of neutral pH, e.g. Dulbecco's Phosphate-Buffered Saline (DPBS), Hank's balanced salts solution (HBSS) is recommended [6].

3. Tooth mounting and preparation:

a) Mounting: mount the complete tooth (root still present; do not use dentin disks) by preference in dental stone (or acrylic resin) for subsequent surface preparation and specimen fabrication, taking care to carefully align the surface of interest (Fig. 4).

b) Create retention, for instance, by preparing two shallow grooves at two opposite root sites for securing tooth in stone throughout specimen fabrication steps.

c) Tooth region of interest should be above level of stone to avoid subsequent contamination during application of adhesive and dental composite.

d) Exposure of dentin at the occlusal surface is recommended unless research design dictates otherwise.

e) Continue specimen preparation 'immediately' after dentin exposure with smear layer preparation, adhesive treatment, etc., to avoid abundant water uptake by the exposed dentin (if needed, short coverage with wet tissue paper is recommended rather than complete immersion in water).

4. Tooth dependency:

a) Use as much as possible a research design balanced by tooth dependency, e.g. $24 \mathrm{~h}, 3 \mathrm{mo}$., 6 mo., $12 \mathrm{mo}$. aqueous storage with random assignment of individual specimens from the same tooth to each of the four storage groups (Fig. 5A). As the micro-specimens are cut prior to the long-term storage, any pre-testing failures that may have occurred should be equally divided 
DENTAL-2881; No. of Pages 11

4

DENTAL MATERIALS XXX (20I6) XXX-XXX

A

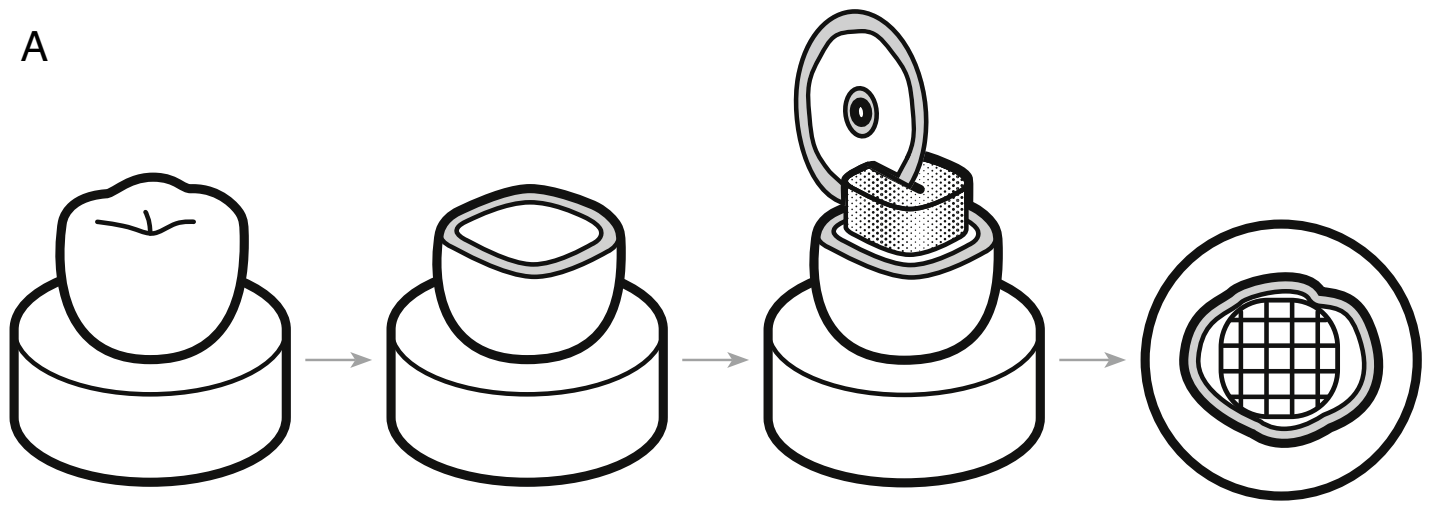

B
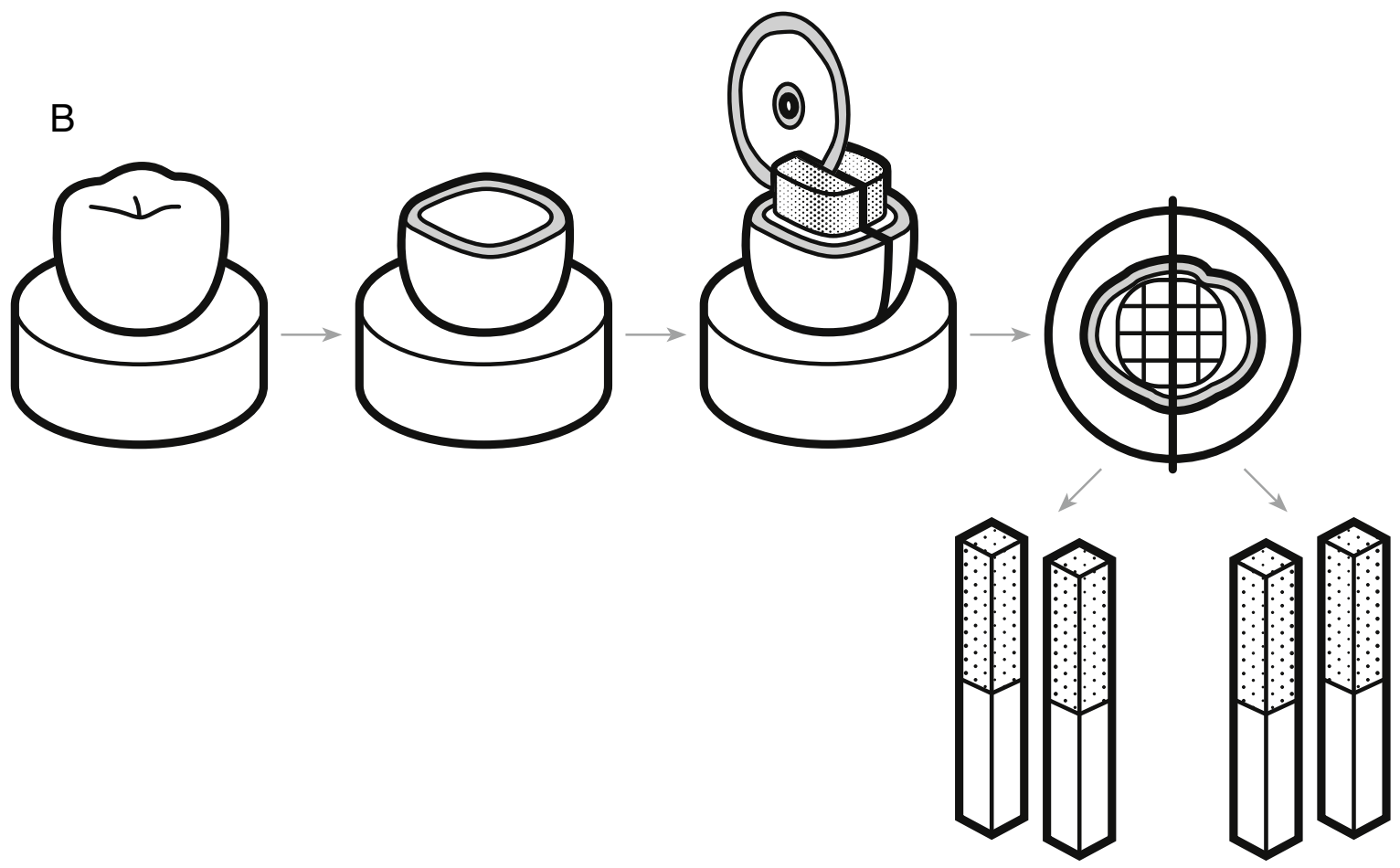

Fig. 5 - Research designs: (A) balanced tooth dependency design with only one adhesive treatment; individual specimens from the same tooth are randomly assigned to each of two, three, or four experimental aging groups. (B) Split-tooth design; each tooth halve serves as a separate experimental group involving a different adhesive application protocol; up to four different adhesive treatments can be applied per tooth, when the tooth surface is separated in 4 quarters.

over the different experimental groups; in case of an odd pt number, one more pt is assigned to the longest storage group.

b) Split-tooth design, e.g. two bonding procedures, with maximum 4 different conditions per tooth: The areas should be separated by a thin and shallow groove (cut using a thin $150-\mu \mathrm{m}$ diamond blade), in which a barvier (ie. razor blade, cover slip) can be positioned to separate both tooth halves (Fig. 5B).

c) Tooth dependency cannot be ignored in data reporting and statistical analyses - see data handling and statistical reporting $[4,7,8]$.
5. Cavity and surface preparation:

a) Cavity preparation: The standard preparation is a flat surface for preparation simplicity and control of variables; however, cavities can also be prepared depending upon the needs of the research design, e.g. study of C-factor, bond to different cavity walls [9].

i) Bonding surfaces tested must be perpendicular to ultimate loading direction.

ii) To simulate bonding to the cavity floor, burs and instruments should be aligned perpendicular to the bonding surface, which may require special equipmont (e.g. MicroSpecimen Former, University of

Please cite this article in press as: Armstrong S, et al. Academy of Dental Materials guidance on in vitro testing of dental composite bonding effec-

tiveness to dentin/enamel using micro-tensile bond strength ( $\mu$ TBS) approach. Dent Mater (2016), http://dx.doi.org/10.1016/j.dental.2016.11.015 
Table 1 - Burs to prepare a bur-cut clinically relevant smear layer.

\begin{tabular}{lll} 
Bur & $(\mu \mathrm{m})$ & \multicolumn{1}{c}{ Notes } \\
\hline 8-fluted carbide & NA & Simulates DIRECT bonding \\
Medium grit diamond & 107 & Simulates DIRECT bonding \\
Fine grit diamond & 46 & Simulates INDIRECT bonding \\
\hline
\end{tabular}

Table 2 - Silicon carbide papers to prepare a clinically relevant smear layer.

\begin{tabular}{lll} 
Paper & \multicolumn{1}{c}{$(\mu \mathrm{m})$} & \multicolumn{1}{c}{ Notes } \\
\hline FEPA P320 & $46.2 \pm 1.5$ & Simulates DIRECT bonding \\
J360 & 48 & \\
ANSI240 & 51 & Simulates INDIRECT bonding \\
FEPA P600 & $25.8 \pm 1$ & \\
J600 & 29 & \\
ANSI360 & 28 & \\
\hline
\end{tabular}

FEPA $\mathrm{P}=$ Federation of European Producers of Abrasives, sanding paper; $\mathrm{J}=$ Japanese Industrial Standard; ANSI = American National Standards Institute.

a P600 recommended by ISO/DTS 11405 for direct bonding.

Iowa). Monitor bur wear and change regularly, e.g. every 6-12 teeth.

b) Smear layer creation:

i) Most appropriate if created immediately after dentin exposure (see above) as well as shortly before bonding.

ii) Burs: Clinically relevant smear layer is best produced by carbide and diamond dental burs that are chosen according to research design (Table 1).

iii) Abrasive papers: Clinically simulated smear layer produced with abrasive papers requires careful attention to align flat surfaces perpendicular to load direction and random surface scratches using a circular motion (rather than in one direction); wet-sanding is preferred (Table 2). Grit sizes are not directly comparable between bur and silicon carbide abrasive paper preparation methods, as different surfaces and smear layers are created.

iv) Other preparation instruments (e.g. laser, sono abrasion, etc.) may be necessary based upon specific research designs, which also need to be employed in an as uniform as possible way.

c) Occlusal depth: 'superficial', by removing last remnant of enamel, to 'mid-coronal' dentin. Check with light microscope and/or 30-40\% phosphoric acid for 3-5 s to confirm enamel removal followed by additional 0.1$\mathrm{mm}$ surface removal.

d) Surface region: to reduce regional and tooth variability, a fixed number of specimens per tooth from a central region of the teeth should be obtained $[4,10]$. For example, four sticks of 2-mm width per tooth for 'trimmed' (dumbbell, hourglass) micro-specimen fabrication (to reach an interface diameter of $0.8-1 \mathrm{~mm} ; 0.8 \mathrm{~mm}$ cylindrical dumbbell has a cross-sectional area of $0.5 \mathrm{~mm}^{2}$ ) (Fig. 6a); 8-16 sticks of 1-mm width per tooth for the 'non-trimmed' technique (Fig. 6b).

6. Adhesive application:
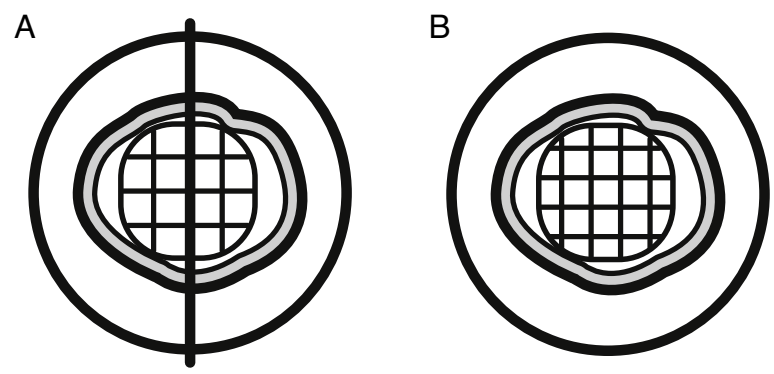

Fig. 6 - Producing a fixed number of micro-specimens with a favorable regional distribution. (A) 4 sticks per tooth for a TRIMMED micro-specimen fabrication; (B) 8-16 sticks per tooth for a NON-TRIMMED micro-specimen fabrication.

a) A detailed description of the application of the adhesive should be provided, as "Applied by manufacturer's directions" is inadequate to reproduce employed methodology; this should include:

i) Conditions in which restorative procedure was completed:

ii) Absolute field control/rubber dam simulation (room conditions): $23 \pm 2{ }^{\circ} \mathrm{C} ; 50 \pm 5 \% \mathrm{RH}$.

iii) Relative field control simulation (mouth conditions): $35 \pm 2{ }^{\circ} \mathrm{C} ; 90 \pm 5 \% \mathrm{RH}$.

iv) Tips and applicators used.

v) Dispensing/mixing.

vi) Time of application.

vii) Method of application (left untouched, actively rubbed, repeated fresh application, etc.).

viii) Air-drying method, pressure, time, etc.

ix) Photopolymerization: see "Photopolymerization" below.

x) Etc.

7. Dental composite:

a) Research design permitting, use same dental composite, with sufficiently high mechanical properties, for all groups and for all studies. An agreed upon industrial-supplied standard dental composite would be a welcome and useful addition to the field. An exception is when one wants to investigate the contribution of the dental composite to the bond strength; then different dental composites should be combined with same adhesive.

b) Provide detailed description of dental composite application (layering, bulk, instrument kind, etc.).

c) Do not exceed recommended increment thickness for specific material, e.g. $2 \mathrm{~mm}$ (unless required by research design, e.g. 4-mm bulk-filling).

d) Apply dental composite without voids or defects. One approach is to apply dental composite directly from compule, then carefully remove "wiped" component of dental composite from tooth and compule tip before photopolymerization and next dental composite application.

e) Build up to reach at least a 4-mm thickness (will serve as support and connection to jig). 

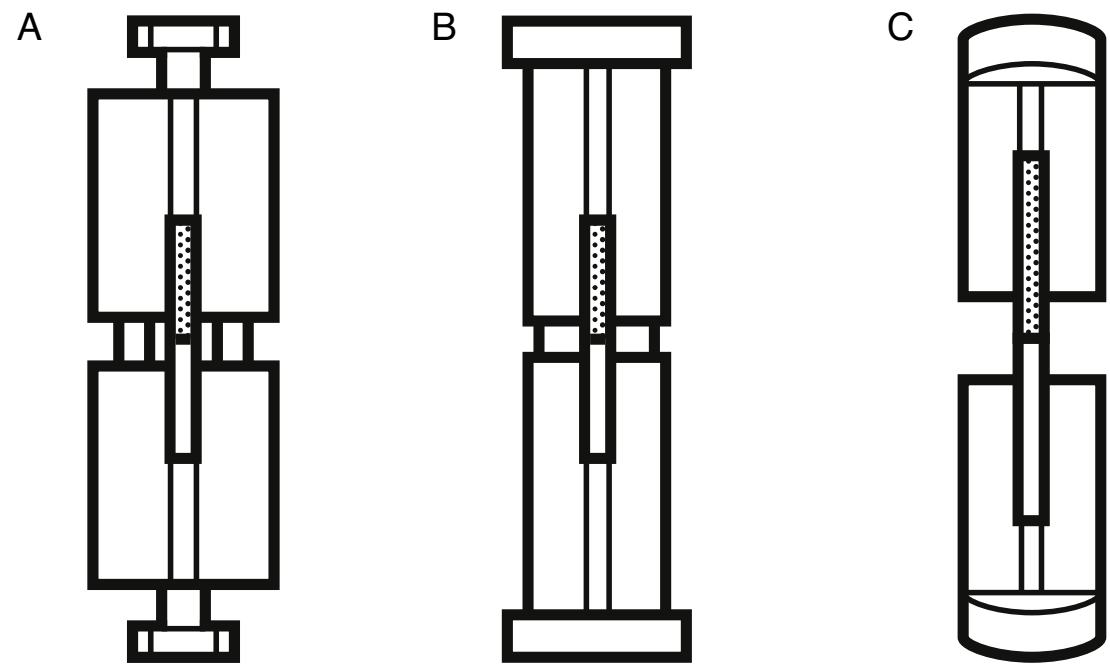

Fig. 7 - ACTIVE gripping devices: (A) notched Geraldeli device; (B) notched Ciucchi's device; (C) Bencor Multi-T notched device.

f) Final layer can be covered with glass plate using light finger pressure before curing.

8. Photopolymerization:

a) Use a curing light that has a radiant exitance from 500 to $2000 \mathrm{~mW} / \mathrm{cm}^{2}$ and deliver sufficient energy to cure materials (adhesive, composite) and report radiant exposure (energy delivered) $\left(\mathrm{J} / \mathrm{cm}^{2}\right)$ to the material surface for each light delivery step [11]. As research design permits, recommend delivering $150 \%$ required incident radiant exposure for adhesive and each dental composite increment to help reduce effect of polymerization as a systematic experimental error.

b) Use photopolymerization unit that matches photoinitiator absorption requirements and report spectral irradiance.

c) Use photopolymerization unit with homogenous irradiance and spectral emission from the photopolymerization tip [12].

d) If possible, use photopolymerization tip that effectively covers entire restoration to avoid necessity of overlapping light curing and report light tip size.

e) Photopolymerization unit is best held in a fixture from a fixed distance perpendicular to the material surface to apply the required radiant exposure. The light tip is kept in the same orientation for all specimens; alternatively, keep tip end parallel with material surface at 1-mm distance for first $5 \mathrm{~s}$ and then let tip end rest on the material surface (plastic protection around tip end needed).

9. Macro-specimen storage/aging (bonded tooth):

Aqueous media aging is better suited to micro-specimens for accelerated aging [13], but can also be performed in the macro-specimen form. Mechanical fatigue and thermomechanical aging are currently best performed in the macro-specimen form.

a) Storage in water or by preference in artificial saliva (see micro-specimen storage/aging below); alternative accelerated aging solutions (as used in literature: propionic acid, acetic acid, ethanol, $\mathrm{NaOCl}$, etc.) could be used, but cannot be considered as standard and therefore should be described in sufficient detail to replicate in future studies. b) Thermo-cycling: Minimum 10,000 cycles [14] (and by preference more) in aqueous media between $5^{\circ} \mathrm{C}$ and $55^{\circ} \mathrm{C}$, starting after $1-7$ day storage in aqueous media at $37^{\circ} \mathrm{C}$. The exposure to each bath should be at least $20 \mathrm{~s}$, and the transfer time between baths should be as short as possible (e.g. 5-10 s). (ISO/DTS 11405 Test type 2 of 500 cycles is inadequate to produce a significant effect.)

c) Mechanical or thermo-mechanical fatigue: Chewing simulator with bonded teeth in single or multiple chambers: use a round 6-mm diameter steatite agonist, apply (for example) 100,000 cycles at $50 \mathrm{~N}$ at a frequency of $0.5 \mathrm{~Hz}$ with (optionally) 2500 simultaneous thermal cycles between $5^{\circ} \mathrm{C}$ and $55^{\circ} \mathrm{C}$ for $30 \mathrm{~s}$ at each temperature [15].

10. MACRO- to MICRO-specimen stick formation by sectioning (Figs. 5 and 6):

a) Diamond saw sectioning: Specify speed, pressure/load, etc.; monitor wear and regularly change blades.

b) Gauge saw sectioning is recommended, that is spacers of desired width between sets of saw blades for single pass sectioning.

c) Reinforce/support sectioned sticks/slabs with dental stone (or alginate or other more rigid impression material) between first and second saw pass.

d) $1 \times 1 \mathrm{~mm}$ maximum size for 'non-trimmed' $\mu$ TBS testing; $2 \times 2 \mathrm{~mm}$ specimen dimensions for 'trimmed' $\mu$ TBS testing.

e) Micro-specimens that de-bond during this sectioning process should be gathered, and recorded as 'pretesting failures'.

f) Diamond wire sectioning has been reported to reduce sectioning defects [16], but has not been commonly used to date.

g) Remaining dentin thickness should be measured for each micro-specimen and recorded for possible influence on resultant bond strength [17]. A minimum of 
A

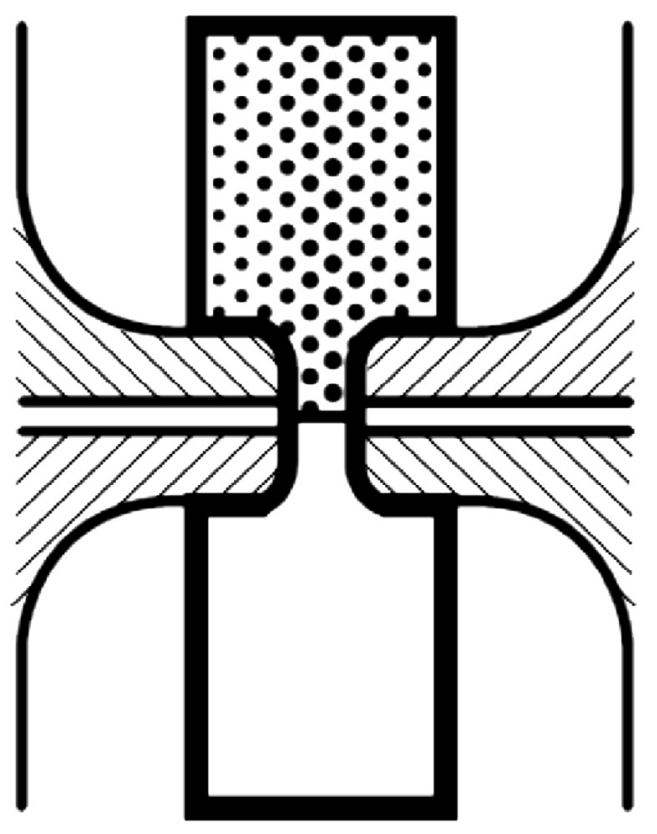

B
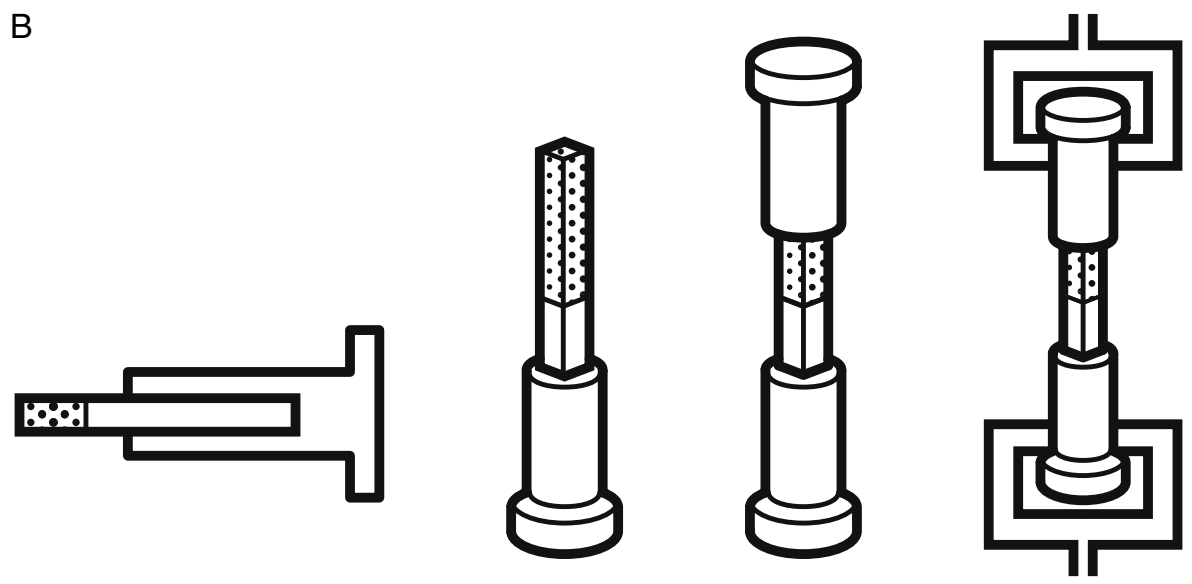

Fig. 8 - Passive gripping devices: (A) Dircks device; (B) semi-passive gripping device using rivets.

$1 \mathrm{~mm}$ remaining dentin thickness should be present for testing.

11. Additional specimen processing to create 'trimmed' (dumbbell, hourglass) micro-specimens:

a) Do not allow the tooth to dehydrate during the sectioning process.

b) Dumbbell recommended over other geometries due to presence of straight gauge region producing uniformly stressed testing area of interest. Fractures that occur outside of this uniformly stressed gauge region are not recommended for direct calculation of mean $\mu$ TBS (ASTM C1273-15 Standard Test Method for Tensile Strength of Monolithic Advanced Ceramics at Ambient Temperatures). c) Circular neck and gauge region formation preferred over sharp edged to avoid stress concentration at corners.

d) Requires larger sticks for trimming, e.g. $2 \times 2 \mathrm{~mm}$ (see above).

e) Requires specialized mechanical or computer-driven equipment for consistent and uniform size and geometry, as well as consistent specimen loading (e.g. MicroSpecimen Former, University of Iowa); preparation by hand is NOT acceptable.

f) Micro-specimens that de-bond during this trimming process should be gathered, and recorded as 'pretesting failures'.

12. Micro-specimen storage/aging (non-trimmed and trimmed sticks): 
a) Aqueous storage (see above for alternative aging solutions)

i) Short-term (<1 month) - aqueous storage media is replaced weekly to avoid contamination.

ii) Medium-term (1-6 months) - aqueous storage media containing $0.5 \%$ Chloramine- $\mathrm{T}$ is replaced biweekly to avoid contamination.

iii) Long-term (6 months or longer) - aqueous storage media containing $0.5 \%$ Chloramine- $\mathrm{T}$ is replaced biweekly to avoid contamination (ISO/DTS 11405 Test Type 3) [5].

b) Thermo-cycling

i) Special containers to hold the micro-specimens are needed to maintain them securely and allow efficient water exchange. Although micro-specimens may show degradative effects more readily than macro-specimens, the thermo-cycling regimen described above is recommended [14].

c) Mechanical fatigue or thermo-mechanical fatigue

i) Four-point bending cyclic loading of individual $\mu$ TBS stick specimens until failure or $10^{6}$ cycles (specify load and fatigue frequency) [18].

ii) Cyclic tensile fatigue loading of micro-specimens fixed by their bottom and top into a material tester that enables cyclic loading [19].

iii) Micro-rotary fatigue of individual $\mu$ TBS dumbbells: specimen rotated at $4 \mathrm{~Hz}$ around their main axis, while being loaded at a fixed distance from the adhesive interface to induce a sinusoidal cycling tensile and compression stress at the outer surface of the adhesive-tooth interface. The specimen is continuously sprayed with water at $37^{\circ} \mathrm{C}$ throughout testing. Specimens stressed until failure or until 100,000 cycles are reached to report a median micro-rotary fatigue resistance [20].

iv) Chewing simulator with adhesive-dentin specimens in multiple chambers: using a round $6 \mathrm{~mm}$ diameter steatite agonist to apply (for example) 100,000 cycles at $20 \mathrm{~N}$ at a frequency of $0.5 \mathrm{~Hz}$ with (optionally) 2500 simultaneous thermal cycles between $5^{\circ} \mathrm{C}$ and $55^{\circ} \mathrm{C}$ for $30 \mathrm{~s}$ at each temperature [15].

13. Specimen gripping device, AKA "jig"

a) Active gripping jigs (Fig. 7):

Active gripping devices should use: (1) some method for careful specimen alignment and (2) a gel-like command-set glue (examples: Zapit, Dental Ventures of America, Corona, CA, USA; Model Repair II Blue, Dentsply-Sankin, Ohtawara, Japan) that permits controlled application sufficiently remote from the adhesive interface and to all sides of the stick (examples shown below). By preference, align the specimen in the soft glue gel prior to the application of the hardener (spray) to cure.

i) Notched Geraldeli's device (Fig. 7A) [21];

ii) Notched Ciucchi's jig (Fig. 7B) [22];

iii) Bencor Multi-T notched device (Fig. 7C) [23];

iv) Top-bottom design [22].

b) Passive gripping jigs (Fig. 8): i) Dircks glue-less self-aligning passive gripping device for dumbbell micro-specimens (Fig. 8A) [24];

ii) Semi-passive gripping device: stick glued in rivets to create a modified pseudo-dumbbell microspecimen that is subsequently passively gripped for testing (Fig. 8B) [25].

14. Actual test:

a) Do not allow the micro-specimens to dehydrate during processing $\left(23 \pm 2{ }^{\circ} \mathrm{C} ; 50 \pm 5 \% \mathrm{RH}\right)$

b) Pre-load force: $0.5-1.0 \mathrm{~N}$.

c) Loading rate: $0.5-1.0 \mathrm{~mm} / \mathrm{min}$, producing final fracture in 5-10 s from reaching pre-load force.

15. Storage and processing of the de-bonded microspecimens:

a) Do not allow the fractured micro-specimens to dehydrate for failure analysis using light-microscopy (LM failure analyses).

b) For 'SEM failure analysis', commonly of selected representative cases (measured bond strength close to mean $\mu$ TBS, low and high values) and of ptf. The microspecimens should be fixed (can be stored for up to $12 \mathrm{~h}$ at $4{ }^{\circ} \mathrm{C}$ in buffered glutaraldehyde-based fixative solution), dehydrated and dried (critical-point or HMDS drying) following common SEM specimen processing.

16. Failure analysis [3]:

a) It is recommended to record the failure pathway using a failure diagram as shown in Fig. 9A.

b) Always analyze both sites (tooth, composite) of the micro-specimen together.

c) LM failure analysis - 2 options:

i) Estimate area-\% per failure mode ('Adhesive at interface', 'Cohesive in Composite', 'Cohesive in bonding', 'Cohesive in dentin') per microspecimen; alternatively, semi-quantitative measurement of area-\% per failure mode using image analysis (time-consuming!).

ii) Categorize each micro-specimen according to its overall failure mode ('Adhesive at interface', 'Cohesive in Composite', 'Cohesive in bonding', 'Cohesive in dentin', 'Mixed involving interfacial de-bonding')

d) SEM failure analysis:

i) Mostly only illustrative, or in case the LM failure analysis does not allow clear interpretation of the failure mode.

17. Data reporting and statistical analysis:

a) Detailed reporting of all steps is critically important for good science. All specimen handling and testing procedures have the potential to effect the resultant data and must be reported.

b) Report details of burs or instruments used for surface preparation (e.g. grit size of diamond bur, settings of laser, etc.).

c) Minimum number of five teeth per experimental/control group.

d) Minimum number of 15 specimens per experimental/control group.

e) If data are normally distributed, report both ANOVA and Weibull distribution. 
A

\section{Dentin}

Dental composite

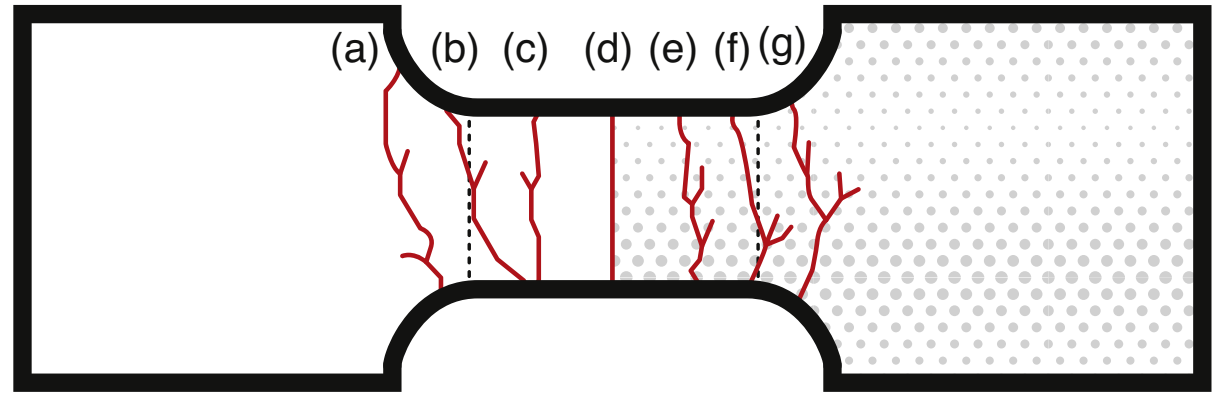

\begin{tabular}{|l|l|}
\hline $\mathbf{a , b}, \mathbf{f} \& \mathbf{g}$ & faliure outside of gauge length (testing region) as shown by dotten lines, right censored data points \\
\hline c & valid test, cohesive faliure in dentin substrate \\
\hline d & valid test, apparently adhesive and mixed adhesive/substrate(s) faliure pathways \\
\hline e & valid test, cohesive faliure in dental composite substrate \\
\hline
\end{tabular}

B

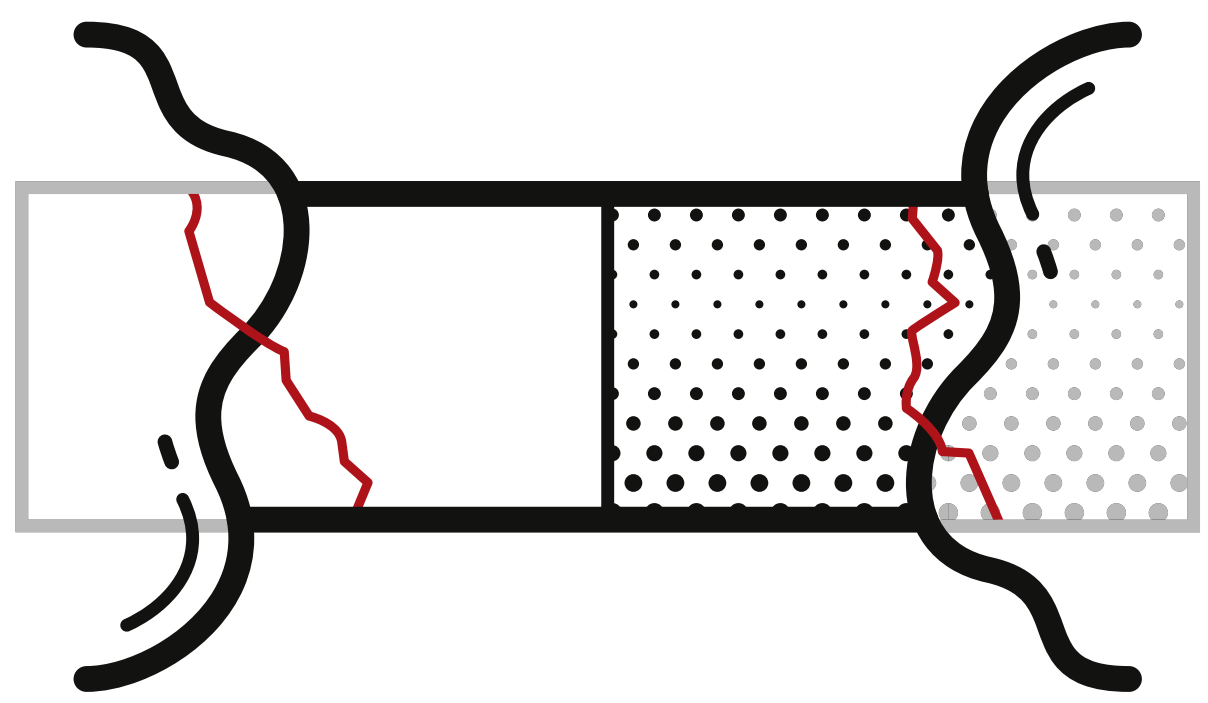

Fig. 9 - (A) Failure pathway determination and recording. (B) Any failure pathway involving glue should be considered an invalid test. The micro-specimen design shown has no designated testing region.

f) Tooth dependency, at a minimum, must be reported as "a researcher who ignores this correlation may be more likely to find statistically significant results when none are present in the data" [8]. How the within tooth correlation of specimens is statistically managed is beyond the scope of this guideline (e.g. random effects with ANOVA; frailty effects with Weibull).

g) 'Manipulation failures' (human errors) should not be included for the mean $\mu$ TBS calculation, but should be explicitly reported.

h) 'Pre-testing failures', including 'aging failures' (debonding during storage or mechanical aging) i) Must be explicitly reported and included in the data sets for the mean $\mu$ TBS calculation [3].

ii) Should be treated as left-censored data and a value should be assigned [26]:

a. $0 \mathrm{MPa}$;

b. Mean between $0 \mathrm{MPa}$ and the lowest measured value in the specific experimental group (or computer-determined random value between $0 \mathrm{MPa}$ and the lowest value measured in the specific experimental group); in case of a spontaneous failure during water storage or 
thermo-cycling (non-mechanical fatigue), $0 \mathrm{MPa}$ can be assigned to the ptf.

i) Tensile testing that produces invalid fracture/debonding pathways

i) Dumbbells: failures due to fracture outside of the defined testing region should be treated as rightcensored data (Fig. 9A).

ii) Sticks: fractures due to de-bonding pathways involving the glue should not be included in the mean $\mu$ TBS calculation, but should be explicitly reported (Fig. 9B).

\section{Guidelines/specific recommendations for adhesive-enamel $\mu$ TBS testing}

Micro-tensile bond strength testing on enamel is more difficult than on dentin due to the relatively brittle nature of enamel. A macro- or micro-shear [27] bond strength test is an alternative approach that requires less specimen processing and thus reduces the possibility of introducing surface or edge defects; therefore, also fewer pre-testing failures are reported [16]. The $\mu$ TBS protocol for adhesive-enamel interface testing is the same as for dentin, except for steps $3 e$ and $4 a, b$, as shown below:

3-enamel. Tooth mounting and preparation for enamel $\mu \mathrm{TBS}$ :

e) Buccal/lingual surface recommended unless research design dictates otherwise.

4-enamel. Tooth dependency for enamel $\mu$ TBS:

a) Use research design balanced by tooth dependency whenever possible, e.g., $24 \mathrm{~h}$ and 6 mo. aqueous storage with random assignment of individual micro-specimens from the same tooth to each of the 2 storage groups.

b) Split-tooth design, e.g. two different conditions per tooth: the areas should be separated by a thin and shallow groove (cut using a thin 150- $\mu \mathrm{m}$ diamond blade), in which a barrier (i.e. razor blade, cover slip) can be positioned to separate both tooth parts; alternatively, 2 different adhesive procedures could be applied to either the buccal or lingual enamel surface.

\section{Acknowledgments}

The authors are grateful to Joshua Cheetham for content contributions, Jan De Munck and Jorge Perdigão for their critical review of the manuscript and the Academy of Dental Materials for support during the development and writing of these guidelines.

\section{REFERENCES}

[1] Roeder L, Pereira PNR, Yamamoto T, Ilie N, Armstrong S, Ferracane J. Spotlight on bond strength testing - unraveling the complexities. Dent Mater 2011;27:1197-203.

[2] Van Meerbeek B, Peumans M, Poitevin A, Mine A, Van Ende A, Neves A, et al. Relationship between bond-strength tests and clinical outcomes. Dent Mater 2010;26:E100-21.

[3] Armstrong S, Geraldeli S, Maia R, Raposo LHA, Soares CJ, Yamagawa J. Adhesion to tooth structure: a critical review of "micro" bond strength test methods. Dent Mater 2010;26:E50-62.

[4] Eckert GJ, Platt JA. A statistical evaluation of microtensile bond strength methodology for dental adhesives. Dent Mater 2007;23:385-91.

[5] ISO/TC $106 \mathrm{D}$, Subcommittee SC1. Filling and restorative materials. ISO/TS 11405:2015, Dentistry-testing of adhesion to tooth structure; 2015.

[6] Habelitz S, Marshall GW, Balooch M, Marshall SJ. Nanoindentation and storage of teeth. J Biomech 2002;35:995-8.

[7] Sattabanasuk V, Vachiramon V, Qian F, Armstrong SR. Resin-dentin bond strength as related to different surface preparation methods. J Dent 2007;35:467-75.

[8] Hannigan A, Lynch CD. Statistical methodology in oral and dental research: pitfalls and recommendations. J Dent 2013;41:385-92.

[9] Van Ende A, De Munck J, Van Landuyt KL, Poitevin A, Peumans M, Van Meerbeek B. Bulk-filling of high C-factor posterior cavities: effect on adhesion to cavity-bottom dentin. Dent Mater 2013;29:269-77.

[10] Shono Y, Ogawa T, Terashita M, Carvalho RM, Pashley EL, Pashley DH. Regional measurement of resin-dentin bonding as an array. J Dent Res 1999;78:699-705.

[11] Platt JA, Price RB. Light curing explored in Halifax. Oper Dent 2014;39:561-3.

[12] Price RBT, Labrie D, Rueggeberg FA, Sullivan B, Kostylev I, Fahey J. Correlation between the beam profile from a curing light and the microhardness of four resins. Dent Mater 2014;30:1345-57.

[13] De Munck J, Van Meerbeek B, Yoshida Y, Inoue S, Vargas M, Suzuki K, et al. Four-year water degradation of total-etch adhesives bonded to dentin. J Dent Res 2003;82:136-40.

[14] Gale MS, Darvell BW. Thermal cycling procedures for laboratory testing of dental restorations. J Dent 1999;27:89-99.

[15] Frankenberger R, Pashley DH, Reich SM, Lohbauer U, Petschelt A, Tay FR. Characterisation of resin-dentine interfaces by compressive cyclic loading. Biomaterials 2005;26:2043-52.

[16] Sadek FT, Monticelli F, Muench A, Ferrari M, Cardoso PEC. A novel method to obtain microtensile specimens minimizing cut flaws. J Biomed Mater Res B 2006;78B:7-14.

[17] Poitevin A, De Munck J, Van Landuyt K, Coutinho E, Peumans M, Lambrechts P, et al. Critical analysis of the influence of different parameters on the microtensile bond strength of adhesives to dentin. J Adhes Dent 2008;10:7-16.

[18] Staninec M, Kim P, Marshall GW, Ritchie RO, Marshall SJ. Fatigue of dentin-composite interfaces with four-point bend. Dent Mater 2008;24:799-803.

[19] Poitevin A, De Munck J, Cardoso MV, Mine A, Peumans M, Lambrechts $\mathrm{P}$, et al. Dynamic versus static bond-strength testing of adhesive interfaces. Dent Mater 2010;26:1068-76.

[20] De Munck J, Braem M, Wevers M, Yoshida Y, Inoue S, Suzuki $\mathrm{K}$, et al. Micro-rotary fatigue of tooth-biomaterial interfaces. Biomaterials 2005;26:1145-53.

[21] Perdigao J, Geraldeli S, Carmo ARP, Dutra HR. In vivo influence of residual moisture on microtensile bond strengths of one-bottle adhesives. J Esthet Restor Dent 2002;14:31-8.

[22] Poitevin A, De Munck J, Van Landuyt K, Coutinho E, Peumans $\mathrm{M}$, Lambrechts $\mathrm{P}$, et al. Influence of three specimen fixation modes on the micro-tensile bond strength of adhesives to dentin. Dent Mater J 2007;26:694-9.

[23] Albaladejo A. In vitro investigations methods of the factors influencing resin-dentin bond durability. Av Odontoestomatol 2008;24:267-76. 
[24] Armstrong SR, Vargas MA, Fang Q, Laffoon JE. Microtensile bond strength of a total-etch 3-step, total-etch 2-step, self-etch 2-step, and a self-etch 1-step dentin bonding system through 15-month water storage. J Adhes Dent 2003;5:47-56.

[25] Lu S, Zhao SJ, Wang WG, Gao Y, Zhang Y, Dou Q, et al. A new fixation method for stick-shaped specimens in microtensile tests: laboratory tests and FEA. J Adhes Dent 2013;15:511-8.
[26] Roulet JF, Van Meerbeek B. Statistics: a nuisance, a tool, or a must? J Adhes Dent 2007;9:287-8.

[27] Beloica M, Goracci C, Carvalho CA, Radovic I, Margvelashvili M, Vulicevic ZR, et al. Microtensile vs microshear bond strength of all-in-one adhesives to unground enamel. J Adhes Dent 2010;12:427-33. 